\title{
A case of successful survival from ventricular free wall micro-rupture without surgery
}

\author{
Jae-Bin Seo, Ki-Hyun Jeon, Jin-Hyun Park, Sang-Hyun Kim, Joo-Hee Zo, Myung-A Kim, \\ Woo-Young Chung ${ }^{*}$ \\ Division of Cardiology, Department of Internal Medicine, Seoul National University Boramae Medical Center, Seoul, Korea \\ Email: *chungwy3023@gmail.com
}

Received 10 January 2013; revised 4 March 2013; accepted 11 April 2013

Copyright (C) 2013 Jae-Bin Seo et al. This is an open access article distributed under the Creative Commons Attribution License, which permits unrestricted use, distribution, and reproduction in any medium, provided the original work is properly cited.

\begin{abstract}
We report a case of successful survival from ventricular free wall rupture without surgery, which was not overt rupture but micro-rupture. The patient was diagnosed with acute myocardial infarction (AMI) and underwent primary percutaneous coronary intervention (PCI). Myocardial blood leakage could be detected on transthoracic echocardiography with intravenous infusion of contrast dye 3 days after PCI. This examination revealed that there was not overt perforation but diffuse fissure. Because of his critical condition, he was treated conservatively without surgery. Nevertheless, he survived eventually.
\end{abstract}

Keywords: Myocardial Infarction; Heart Rupture; Echocardiography

\section{INTRODUCTION}

Ventricular free wall rupture is a disastrous complication of acute myocardial infarction (AMI). Rupture of ventricular free wall occurs in 0.8 percent to 6.2 percent of AMI patients and is a major cause of in-hospital death for ST segment elevation myocardial infarction (STEMI) [1]. Also, it happens after traumatic chest injury [2]. Usually, the diagnosis is made by two-dimensional echocardiography and the treatment of choice is surgical correction. We recently encountered a patient with ventricular free wall rupture after AMI who survived without surgery.

\section{CASE REPORT}

A 65-year-old male patient visited the emergency department due to chest pain of abrupt onset. He was a current smoker. The initial ECG showed an inferior wall

${ }^{*}$ Corresponding author.
ST elevation and initial cardiac enzyme levels were within normal limits. However, 5 hours after arrival, CK level was $42 \mathrm{IU} / \mathrm{L}$ and Troponin-I was positive. Therefore, he underwent emergent coronary angiography. During the procedure, heart rate decreased to 48 beats per minute on the ECG, which made us implant a temporary pacemaker. Coronary angiography showed total thrombotic occlusion of the proximal right coronary artery (RCA) (Figure 1), discrete luminal stenosis of proximal left anterior descending artery (LAD) up to $50 \%$, and diffuse stenosis of distal left circumflex artery (LCx) up to $50 \%$. Following diagnostic coronary angiography, PCI for RCA total occlusion was performed. After insertion of temporary pacemaker and intravenous (IV) infusion of abxicimab, thrombus was removed by aspiration catheter, and plain old balloon angioplasty (POBA) was performed using $3 \times 20 \mathrm{~mm}$ Ryujin balloon. Thereafter, residual stenosis was about $70 \%$. Then, a $3 \times 28 \mathrm{~mm}$

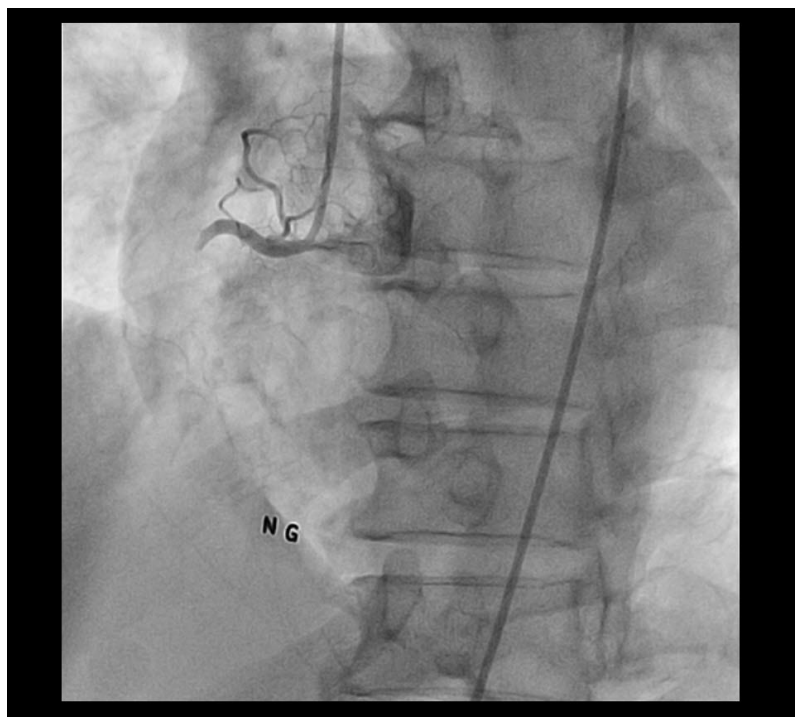

Figure 1. Thrombotic occlusion of proximal RCA. 
Cypher stent was implanted successfully. Although final angiography showed trapped thrombi in the stent, antegrade flow grade was TIMI 3 (Figure 2) and we decided to make an end of the procedure and start IV abxicimab infusion for 12 hours after the sheath removal.

Early in the next morning, shock with decreased urine output was developed. Despite the support of inotropics, he remained in shock state. Transthoracic echocardiographic examination demonstrated cardiac tamponade without definite myocardial rupture (Figure 3). After confirming cardiac tamponade, emergent pericardiocentesis (PCC) was performed. On relook coronary angiography, we could not find any potential cause of cardiac tamponade including coronary arterial perforation (Figure 4). The day after PCC, we could not find pericardial effusion on the echocardiographic examination and the drainage amount via PCC was about 55 cc for 24 hours.

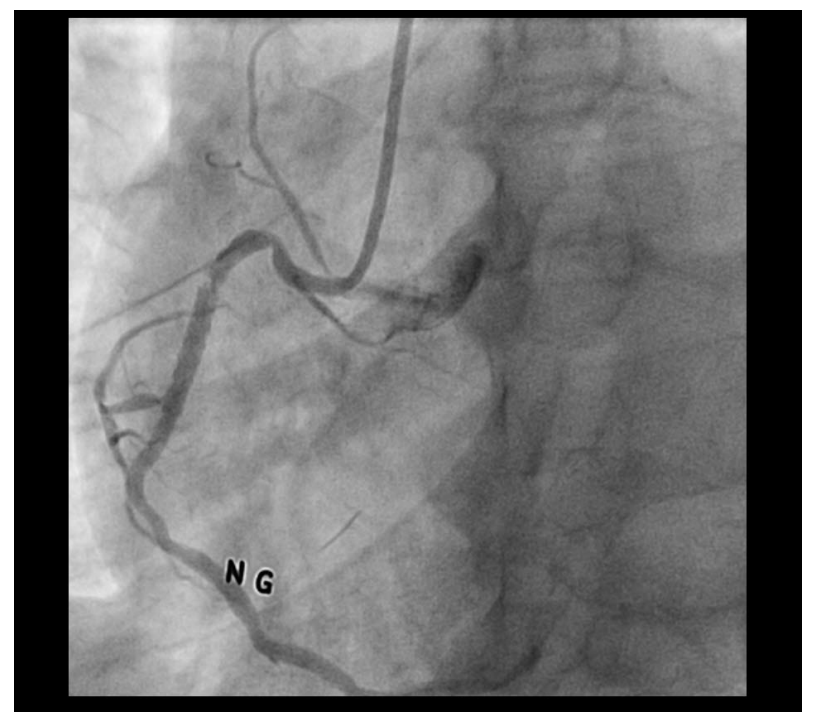

Figure 2. Trapped thrombi in RCA stent.

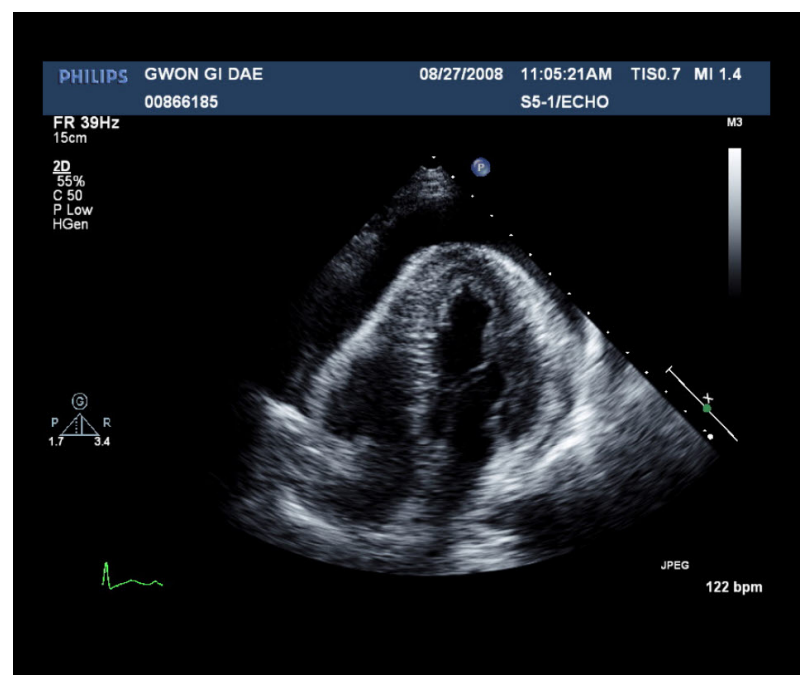

Figure 3. Large amount of pericardial effusion.

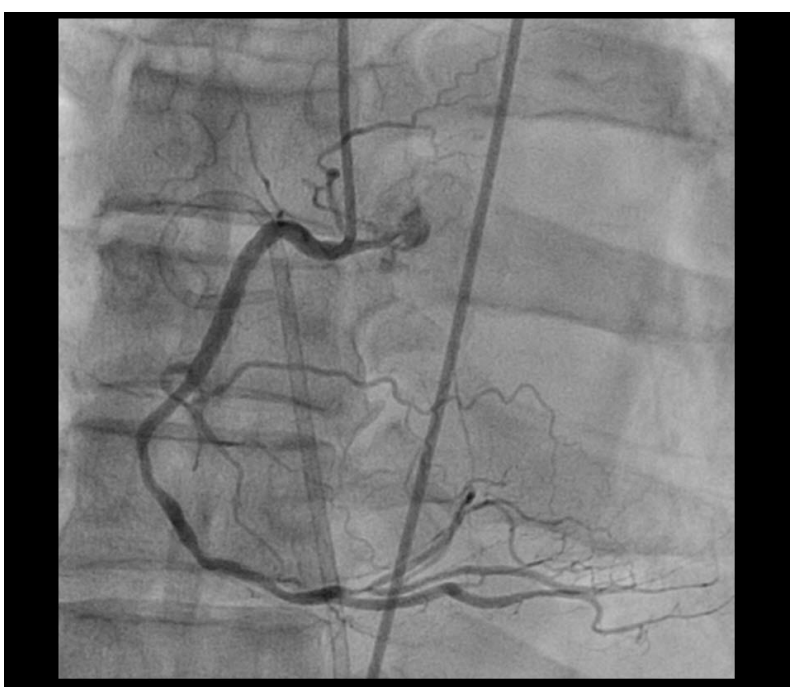

Figure 4. No stenosis in RCA stent.

Two days after PCC, cardiac arrest was developed after an abrupt decrease in blood pressure. Cardiopulmonary resuscitation (CPR) was performed immediately. At the same time, the drainage amount of PCC increased abruptly. Echocardiography showed redevelopment of pericardial effusion and severely depressed RV systolic function. However, once again, we failed to detect any myocardial rupture site. Instead, we observed abnormal movement of the RV free wall. It seemed that there was asynchronous movement between mid ventricular level and apical level of myocardium. Even with agitated saline test, no blood leakage around this point was observed on two-dimensional echocardiography. Finally, we injected radiocontrast (Iodixanol, Visipaque $\left.{ }^{(}\right)$) via an antecubital vein and found diffuse leakage of contrast through the RV free wall (Figure 5). However, we did not perform another pericardiocentesis because the drainage was good and decrease of pericardial effusion was demonstrated on subsequent echocardiographic examinations. Considering the fact that the patient was unstable and we could not find a definite rupture site, operation could not be performed. Fortunately, the drainage amount of PCC got decreased afterwards without reaccumulation of pericardial effusion on serial echocardiographic examinations. Twenty days later, a follow-up echocardiographic examination showed just focal hypokinesia of inferior wall and RV free wall and no pericardial effusion was found. He could be discharged on day 42 and, currently, he is doing well without chest pain or dyspnea.

\section{DISCUSSION}

Ventricular free wall rupture after acute myocardial infarction is a catastrophic condition. The free wall rupture usually leads to hemopericardium, cardiac tamponade 


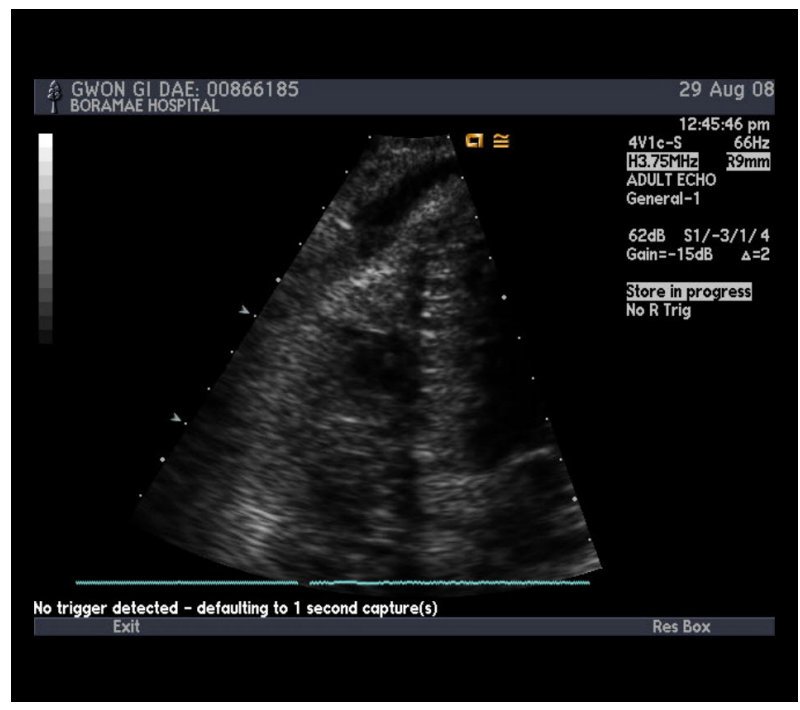

Figure 5. The passage of contrast dye into pericardial space.

and death. Patients' survival depends on the early detection, hemodynamic stabilization and especially prompt surgical intervention.

In the case of our patient, there was a shock from cardiac tamponade on the next morning after PCI. However, on two-dimensional echocardiography, we could not find any demonstrable rupture site or color jet flow. We thought that free wall rupture, pacemaker tip injury, post infarction pericarditis and arterial perforation might be potential cause of pericardial effusion. First, the possibility of free wall rupture was not high, because there was no evidence for communication between the ventricle and pericardial space on echocardiographic examination. Second, although the pacemaker lead tip moved slightly in concordance with heart beat during PCI, we were convinced it was minimal and not traumatic. Third, post infarction pericarditis was not well consistent with his fulminant course. Fourth, on index PCI procedure and relook coronary angiography, no arterial perforation was detected. Hence, we could not elucidate definite cause of pericardial effusion at that time.

Ventricular free wall rupture could be diagnosed by pericardiocentesis, echocardiography, or cardiac catheterization, depending on the situation [1]. Contrast echocardiography has been used to detect intracardiac shunts, enhance Doppler signals, opacify left ventricle, or assess myocardial perfusion [3]. Furthermore, it is also useful to detect myocardial rupture. Mittle S. et al. described two cases using contrast echocardiography in the assessment of myocardial rupture [4]. In the first case, contrast echocardiography did not show characteristic echocardiographic images, making them confident in excluding the diagnosis of myocardial rupture. In the second case, the examination revealed leakage of contrast into the pericardial space. Wilkenshoff U.M. et al. reported a case where incomplete myocardial rupture with inferior infarction was detected by contrast echocardiography [5]. The echocardiography showed pericardial effusion, abnormal inferior wall motion and myocardial irregularities of inferior wall. Myocardial rupture was confirmed by the clear delineation using contrast echocardiography. The patient underwent immediate surgical repair, leading to a favorable long-term outcome. In addition, Trindade M.L. et al. recently demonstrated a case where left ventricular free wall rupture was diagnosed early by contrast echocardiography [6].

In our case, routine echocardiographic examination did not show overt perforation of ventricular free wall rupture. However, contrast echocardiography demonstrated sequential opacification of right ventricle and pericardial space. Therefore, it would be reasonable to assume that there was not overt perforation but diffuse fissure or micro-ruptures in myocardium, through which blood could leak and ooze. IV abciximab infusion might interfere with the healing process of diffuse myocardial fissure. However, 2 days after discontinuation of IV abxicimab, we were faced up with abrupt increase of pericardial effusion.

There are two types of contrast agents for echocardiography [3]. One type is to opacify the right heart and the other is to opacify the left and myocardium. Agitated saline is a representative contrast for right heart. The contrast agents used used by Mittle S. et al., Wilkenshoff U.M. et al. and Trindade M.L. et al. were Optison or PESDA categorized as left heart contrast. We failed to demonstrate the connection of right ventricle and pericardial space with agitated saline. Instead, with iodixanol, we could infer the communication between two spaces. Generally, agitated saline could not traverse the pulmonary capillary bed. Considering this fact, the width of diffuse fissure would be smaller than that of the pulmonary capillary bed.

Both left and right ventricular free wall rupture occur with similar presentation and pathophysiology. Therefore, the treatment could be discussed together [7]. Medical therapy is considered to be a bridge to surgery and surgical repair needs to be carried out as soon as possible. Figueras J. et al. carried out prospective evaluation of the medical management of patients with clinically suspected free wall rupture after acute myocardial infarction [8]. Of the 141 patients, 36 patients could be discharged without surgical treatment in that study. They suggested a conservative management in patients with important comorbidity factors that greatly increase the surgical risk such as severe chronic lung disease, renal failure, extensive myocardial infarction, or serious peripheral vascular disease. However, this strategy represents a challenge in most settings.

In the case of our patients, surgery was not undertaken 
in agreement with the surgical team. The calculated logistic Euroscore was more than 70 percent, which suggested extremely high risk surgery [9]. Moreover, even though we could choose surgical treatment, it was not obvious how much and which portion of RV should be resected. Of course, heart transplantation might be a good option. However, it was hardly possible to find a donor urgently in Korea. Therefore, we decided to take supportive care and fortunately the patient recovered.

In conclusion, it is significant to note 3 points: 1 ) Myocardial blood leakage through diffuse fissure, or micro-rupture, could occur on the early stage of AMI. 2) Myocardial blood leakage through right ventricular free wall could be detected on transthoracic echocardiogramphy using intravenous infusion of contrast dye. 3) If there is no overt perforation, and micro-rupture is suspected, intensive supportive care could be the better alternative to surgical approach.

\section{REFERENCES}

[1] Antman, E.M. (2007) ST-elevation myocardial infarction: Management. In: Libby, P., Robert, O.B., Douglas, L.M. and Zipes, D.P., Eds., Braundwald's Heart Disease: A Textbook of Cardiovascular Medicine, 8th Edition, Saunders, Philadelphia, 1272-1273.

[2] Seo, J.B., Cho, S.H., Yoon, C.H., et al. (2004) A patient with prior history of open heart surgery, who survived from a traumatic ventricular free wall and coronary artery rupture, without surgical repair. Korean Circulation Jour- nal, 34, 104-106.

[3] Otto, C.M. (2004) Contrast echocardiography. Textbook of Clinical Echocardiography, 3rd Edition, Saunders, Philadelphia, 97-100.

[4] Mittle, S., Makaryus, A.N. and Mangion, J. (2003) Role of contrast echocardiography in the assessment of myocardial rupture. Echocardiography, 20, 77-81. doi:10.1046/j.1540-8175.2003.00012.x

[5] Wilkenshoff, U.M., Ale Abaei, A., Kuersten, B., et al. (2004) Contrast echocardiography for detection of incomeplete rupture of the left ventricle after acute myocardial infarction. Zeitschrift fur Kardiologie, 93, 624-629.

[6] Trindade, M.L., Tsutsui, J.M., Rodrigues, A.C., et al. (2006) Left ventricular free wall impeding rupture in post-myocardial infarction period diagnosed by myocardial contrast echocardiography: Case report. Cardiovascular Ultrasound, 4, 7. doi:10.1186/1476-7120-4-7

[7] Birnbaum, Y., Chamoun, A.J., Anzuini, A., Lick, S.D., Ahmad, M. and Uretsky, B.F. (2003) Ventricular free wall rupture following acute myocardial infarction. Coronary Artery Disease, 14, 463-470. doi:10.1097/00019501-200309000-00008

[8] Figueras, J., Cortadellas, J. and Soler-Soler, J. (2000) Left ventricular free wall rupture: Clinical presentation and management. Heart, 83, 499-504. doi:10.1136/heart.83.5.499

[9] Roques, F., Michel, P., Goldstone, A.R. and Nashef, S.A. (2003) The logistic EuroSCORE. European Heart Journal, 24, 881-882. doi:10.1016/S0195-668X(02)00799-6 